
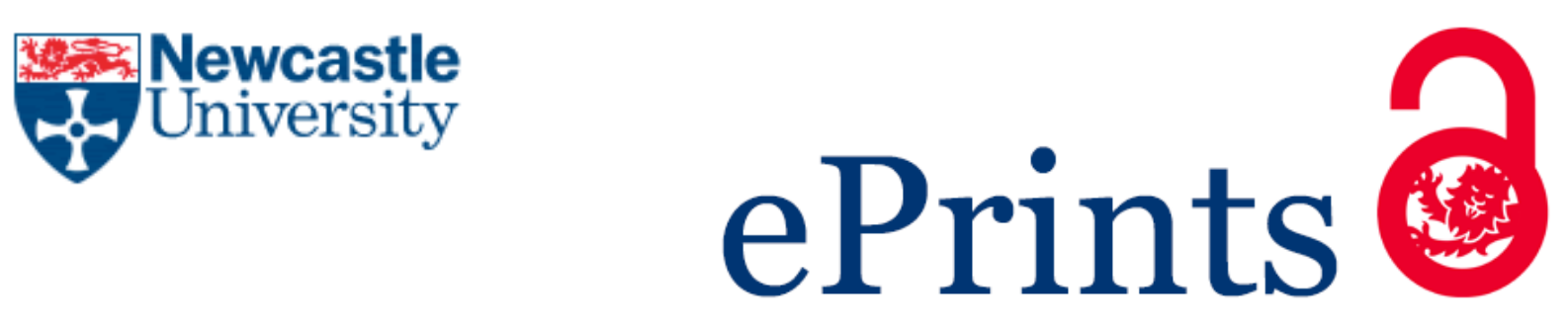

Smith SL, Li L, Joyce T. Engineering of a multi-station shoulder simulator. Proceedings of the Institution of Mechanical Engineers, Part H: Journal of Engineering in Medicine 2016, 230(5), 470-480.

Copyright:

(c) The authors 2016.

DOI link to article:

http://dx.doi.org/10.1177/0954411915611161

Date deposited:

$24 / 05 / 2016$

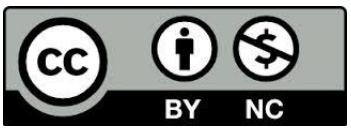

This work is licensed under a Creative Commons Attribution-NonCommercial 3.0 Unported License 


\section{Engineering of a multi-station shoulder simulator}

\section{Simon L Smith, Lisa Li and Thomas J Joyce}

School of Mechanical and Systems Engineering

Newcastle University

Claremont Road

Newcastle upon Tyne

NE1 7RU

England

UK

E-mail: adobewombat@hotmail.com

Telephone: +447913663034

Keywords: simulator, wear, shoulder, validation, CoCrMo, UHMWPE 


\begin{abstract}
This work aimed to engineer a multi-station shoulder simulator in order to wear test shoulder prostheses using recognized shoulder activities of daily living (ADLs). A bespoke simulator was designed, built and subject to commissioning trials before a first wear test was conducted. Five JRI Orthopaedics Reverse Shoulder VAIOS 42mm prostheses were tested for 2.0 million cycles and a mean wear rate and standard deviation of $14.2 \pm 2.1 \mathrm{~mm}^{3} / 10^{6}$ cycles measured for the polymeric glenoid components. This result when adjusted for prostheses diameters and test conditions, showed excellent agreement with results from hip simulator studies of similar materials in a lubricant of bovine serum. The Newcastle Shoulder Simulator is the first multi-station shoulder simulator capable of applying physiological motion and loading for typical ADLs.
\end{abstract}




\section{Introduction to joint simulator design}

Failure of artificial hip joints has long been attributed to polyethylene wear debris $\mathbf{1 , 2}$, and the body's reaction to Ultra-High Molecular Weight Polyethylene (UHMWPE) wear debris is now widely regarded as a limitation to long term artificial joint survival ${ }^{3}$. Similarly, loosening and failure of shoulder implants ${ }^{\mathbf{4 , 5}}$ in vivo has also been identified to be related to wear of the polymeric components.

Shoulder joint replacement is now the third most common orthopaedic procedure after hip and knee replacement, as recorded by the National Joint Registry (NJR) for England, Wales and Northern Ireland ${ }^{\mathbf{6}}$, and the number of procedures is growing rapidly. The numbers of shoulder prostheses implanted and reported to the NJR for comparable periods in $2012^{7}$ and $2013^{6}$ increased dramatically from 2,561 to 4,906.

Despite the popularity of shoulder joint replacements and acknowledgment that wear is a limitation to long term survival, relatively few in vitro shoulder wear studies have been published. Shoulder joints are subject to a number of activities of daily living (ADLs) with various and wide ranging loads and patterns of displacement ${ }^{8}$. The simulators used to test shoulder prostheses have included modified hip ${ }^{\mathbf{9}, 10}$ and knee ${ }^{11}$ simulators, and single station machines ${ }^{\mathbf{1 2}-15}$, and none of the simulator tests have applied full physiological motion and loading. A need therefore exists for a shoulder wear simulator which can apply full physiological motion and loading to replicate a range of ADL's. 
Various national and international standards have been established in an attempt to allow comparison of one wear test to another by defining the test conditions.

Unfortunately, standards do not exist for the wear testing of shoulder joints, which is the aim of this simulator. ISO $14242-1^{16}$ defines displacement and load patterns for hip testing, with ranges typical of physiological patterns ${ }^{17}$ but they are simplified patterns of sinusoidal form rather than physiological. These simplified patterns are used as they have been shown to give wear results comparable to wear results from physiological patterns ${ }^{18,19}$. Hence, a shoulder wear simulator should at least be capable of ranges of motion for physiological patterns, if not capable of the actual physiological patterns.

ISO 14242-1 also defines the environmental conditions under which wear testing of hip joints should be performed such as the temperature and lubricating fluid to be used, and the cycle frequency and duration of testing. ISO $14242-2^{20}$ defines the methods of measurement for such wear testing of hip joints. Again, in the absence of shoulder joint wear test standards, these can provide guidance on appropriate testing. In the interests of obtaining statistically relevant results, wear simulators should allow testing of multiple specimens in a time period that makes the results useful to the design progression of replacement joints. Hence, wear simulators should be multi-station and run reliably for extensive periods of testing.

The basic design specification is now established. The simulator should be multistation, reliable, and at least capable of applying physiological shoulder ranges of motion and load under conditions based on existing protocols for wear testing. Engineering design is often a compromise of conflicting parameters and the design of 
the shoulder simulator is typical in having such confliction. For example, it must be strong and stiff enough to wear test reliably over millions of cycles, and yet mass and consequently inertia of a large number of moving parts should be minimized. Finally, in common with machines in general, simulators ideally should be inexpensive to manufacture, operate and maintain. 


\section{Conceptual design of the Newcastle Shoulder Wear Simulator}

The protocols to measure wear of the shoulder prostheses will be based on ISO-142422. This standard defines both volumetric and gravimetric measurement protocols. The gravimetric protocol requires a loaded, non-articulating station to allow for fluid sorption. Hence, the simulator will require such a station in addition to the articulating and loaded stations.

The simulator should at least be capable of ranges of displacement and load comparable with those observed physiologically in shoulders. Displacement and load patterns defined for wear testing of hips are based on walking, whereas shoulder joints offer the greatest mobility of all the human joints. Motions of the glenohumeral joint during common activities have been reported ${ }^{\mathbf{8}}$, the loading across the joint modelled ${ }^{\mathbf{2 1}}$ and typical activities of daily living have been modelled for shoulder prostheses ${ }^{\mathbf{2 2}}$. Therefore, the simulator must be programmable with the provision for testing these ADL cycles as the various effects on wear are currently unknown. Similarly, the simulator needs to be programmable to have the capability to run tests in accordance with any future standards for wear testing of shoulder joints.

The simulator will have many components including a strong rigid frame and many moving parts. Various materials were considered from which to manufacture all of the components, and the selection criteria included parameters such as weight, cost, ease of manufacture, wear and corrosion properties. The final material selection for many components was aluminium as a best compromise of all the parameters considered. 
Other decisions on the use of materials were as follows. ISO 14242-2 states the prostheses' articulating surfaces must be immersed in a test medium of bovine serum. Any metallic component on the simulator that would be immersed or highly likely to come into contact with bovine serum would be made of stainless steel grade A4 to minimize the highly corrosive effect of the serum. Stainless steel of grade A2 is cheaper and more commonly used, however in the authors' experience it is not sufficiently resistant to corrosion for prolonged exposure to bovine serum. Titanium alloys and those based on Cobalt and Chromium were also considered but discounted due primarily to their high cost compared with A4 stainless steel. Any polymer components likely to be in contact with the serum should be made of a different polymer to that under test, frequently UHMWPE. This decision is based on the opportunity to analyse wear debris from the lubricant in which the joint is being tested. Therefore, any extraneous polymer debris should be identifiable from the joint debris.

Retention of the bovine serum lubricant around the prostheses under test is one of the fundamental considerations of the design. The authors' experience with simulators using flexible gaiters is that gaiters eventually split when subject to millions of testing cycles. This leads to loss of lubricant which in turn can cause fluid starvation of the articulating surfaces and subsequent failure of the joint and ultimately the wear test. Experience with lubricant baths is such that exposed baths can suffer from undesirable contamination of the lubricant and lubricant evaporation, which can also lead to fluid starvation of the articulating interface. Automated methods to maintain lubricant level add cost and complexity, and failure of such systems can lead to failure of the joint and 
wear test. A conceptual solution is to use a lubricant bath in which one part of the joint is mounted and the bath subject to rotational motion. A sectional lid could then largely enclose the bath reducing the risk of contamination and excessive loss and evaporation of the lubricant, whilst still allowing large ranges of motion.

A requirement of ISO $14242-1$ is to maintain the lubricant at $37^{\circ} \mathrm{C} \pm 2^{\circ} \mathrm{C}$. This is can be achieved in various ways, for example with heaters in the lubricant baths, or by enclosing the baths or even the whole simulator and maintaining the enclosed environment at the specified temperature. However, wear testing at approximately $37^{\circ} \mathrm{C}$ has been shown to cause increased protein precipitation which adheres to the articulating surfaces reducing the wear rate ${ }^{23}$. Furthermore, not heating the bovine serum results in less evaporation, less protein precipitation and reduced microbial growth, thus maintaining experimental conditions and critically wear results are similar to clinical results for wear factors, size and shape of the wear debris, polished appearance of the worn polyethylene and undamaged counterface ${ }^{24}$. Not heating the serum also reduces the complexity of the design, the number of components, cost to build and cost to run the simulator. The conceptual designer must make a decision, and the argument not to heat the lubricant is powerful.

An important consideration is how to power and control motion and loading of the simulator. Hydraulics, pneumatics, electrical motors and actuators were considered and existing non-shoulder simulators offer examples of these. Hip ${ }^{25,26}$ and $\mathrm{knee}^{27}$ simulators have successfully used hydraulic motion and loading systems. The not inconsiderable disadvantages of using hydraulics include the capital and running costs, noise, and 
possible contamination of test specimens upon hydraulic leaks. DC stepper motors have been used to power the motions of a simulator ${ }^{28}$, as have AC stepper motors ${ }^{29}$, both allowing accurate and programmable control of the motion cycles as a cheaper solution to hydraulic systems. A single AC motor has also been used with a mechanical linkage ${ }^{30}$ to allow inexpensive and accurate motion cycle delivery and control. Whilst this provides a very cost effective solution, it is rather inflexible when change of the motion cycles is required.

Whilst pneumatic loading has been widely used on many simulators ${ }^{\mathbf{2 8 - 3 2}}$, pneumatic powered motion is less common despite having been proven in knee and finger simulators $^{31,32}$. Pneumatic systems are inexpensive, lightweight, and highly controllable. In many research and industrial sites compressed air is also readily available. As a conceptual solution for motion and loading, pneumatics would seem eminently sensible. Good engineering design must be applied, as ever, to often conflicting parameters such as minimizing the volume of air in the system and air consumption whilst providing sufficient flow for cylinder motion and loading.

The application of motion and loading to the joints under test should be consistent. This is ideally done by a method that inherently ensures that this it is identical across all the joints, for example through rigid drive bars common to all stations. Designs which feature multiple actuators to provide a particular motion should be avoided where possible. Whilst these designs can be calibrated, controlled and checked, the possibility exists for inconsistencies between stations. 
An inherent problem with wear test simulators is wear of the simulator itself. The conceptual design therefore can include large safety factors in the design and selection of bearings and load bearing components. The conceptual designer must consider maintenance of the simulator and the need to replace or repair components as necessary or as part of a routine maintenance schedule. The design should also consider the consequences of component failure and where possible the simulator design should fail safe to protect the joints under test. Protecting the simulator from further damage, operators or other persons, should also be considered and implemented as appropriate. 


\section{Final design of the Newcastle Shoulder Wear Simulator}

The Newcastle Shoulder Wear Simulator, shown in Figure 1, is largely constructed from aluminium with programmable pneumatic motion and loading. It features five articulating and loading stations spaced at $120 \mathrm{~mm}$ between centres, housed in a frame with overall dimensions of $1.0 \mathrm{~m}$ wide, $0.5 \mathrm{~m}$ deep and $0.8 \mathrm{~m}$ tall. A sixth dynamically loaded but non-articulating control station, shown in Figure 2, sits in an aluminium frame $0.25 \mathrm{~m}$ wide, $0.3 \mathrm{~m}$ deep and $0.5 \mathrm{~m}$ tall. Total and reverse shoulder prosthesis designs can both be accommodated and both mounted either anatomically or inverted, as required. However, typically the polymeric components would be mounted below the metallic components in a lubricant bath to ensure the articulating surfaces are lubricated.

[Insert Figure 1.]

[Insert Figure 2.]

When the metallic components are mounted in the upper position, they are subject to abduction-adduction and flexion-extension. The shoulder simulator can apply up to $110^{\circ}$ of motion in the flexion-extension and $90^{\circ}$ in the abduction-adduction axis. If required, it is possible to effectively swap these axes to allow $110^{\circ}$ of motion in the abduction-adduction axis and $90^{\circ}$ in the flexion-extension axis through different mounting of the joint and programmable motion application. The polymeric joint components are mounted in lubricant baths which are subject to internal-external 
rotation axis of up to $90^{\circ}$. Dynamic loading of up to $1,500 \mathrm{~N}$ can be applied. The arrangement of the axes and motions is shown in Figure 3.

\section{[Insert Figure 3.]}

The lubricant baths are bespoke items for the joints under test and the space available in the simulator for the baths was selected to accommodate all currently available shoulder prostheses. Mounting the joints in bespoke baths allows for accurate, repeat mounting of the components. Each bath is covered by a two piece lid, which allows for a large range of motion of the upper metallic component whilst reducing the risk and amount of lubricant contamination and evaporation. The baths used in all six stations are of identical design. Bovine serum of any required dilution or protein content can be used in the baths. Figure 4 shows one of the lubricant baths (a) used in the first wear test of a reverse shoulder prosthesis and the footprint can be seen in which the polymeric component sits. The polymeric component (b) can also be seen in Figure 4 as can the metallic glenosphere component (c). A polymeric retaining ring (d) used to clamp the polymeric test component in position is also shown, and the two part lubricant bath cover (e), which minimizes contamination and evaporation.

[Insert Figure 4.]

Motion of the five articulating stations is by three Norgren pneumatic cylinders with integral position encoders to move the prostheses simultaneously in the flexionextension (Norgren PSA/182063/F1/125), abduction-adduction (Norgren PSA/182050/F1/125), and internal-external rotation (Norgren PSA/182040/F1/125) 
axes. Drive in each axis from the pneumatic cylinders to the stations is via common drive systems to ensure identical motion of the joints under test. ANSYS finite element analysis software allowed the strength to weight ratio of individual frame and drive components to be analysed and optimised during the design process.

Axial loading is applied using a SMC pneumatic cylinder (CP96SDB63-50) for each of the five articulating stations and the sixth non-articulating station, the compressed air supplied from a SMC proportional valve (ITV2050-31F3N) via a manifold. The axial loading cylinders are double acting and back pressure acts as an air spring to remove loading, the air supplied via a manifold to ensure equality of pressure across the six cylinders. The simulator uses two National Instrument controllers (NI USB-6211) programmed in LabView to control the pneumatic system. Closed loop feedback is used for the three motion axes and open loop feedback is used to control the loading, which has been demonstrated to be highly reliable ${ }^{\mathbf{2 8 , 3 0}}$. The LabView programme provides the facility to record the motion and loading data for each cycle. The simulator can be programmed to reproduce individual ADL's and if required a combination of these ADL's to simulate a 'typical' days ADL's. 


\section{Commissioning trials and first wear test}

Commissioning trials are required to ensure that the simulator components and systems, and the operational processes, are as required by the designer, prior to beginning the first wear test. The commissioning trials are essentially a wear test performed as the designer originally specified in all respects. This includes all cleaning and measurement protocols, assembly of test components, calibration and set up of the simulator and its systems, and running of a wear test. The process is essential to finding possible faults in the design and processes, some of which may only become evident as components suffer fatigue failure. Where necessary, procedures can be rewritten, components redesigned, and any other changes made so that the simulator meets the design specification and is subsequently ready to commence actual wear testing.

Calibration of the angular position and motion patterns of the prostheses involved both static and dynamic calibration of the position encoders integral to each of the pneumatic cylinders with actual position of the prostheses components. The position encoders could then be used for closed loop feedback. Loading calibration included calibration of the simulator load cell prior to calibration of the loading of the prostheses components. The latter involved calibrating the load cell measurements with the pressure transducer integral to the pneumatic proportional valve. The pressure transducer could then be used for open loop feedback of the loading cycle.

During commissioning trials, the most rigorous of all the ADLs in terms of greatest motion ranges and loads as ranked by a previous study ${ }^{8}$, was selected to assess the 
performance of the simulator under these demanding conditions. The ADL selected was lifting a $0.5 \mathrm{~kg}$ mass from waist height to head height at a frequency of $1 \mathrm{~Hz}$. Motion ranges per cycle were typically $0^{\circ}$ to $+44^{\circ}$ in flexion-extension, $-53^{\circ}$ to $-5^{\circ}$ in abductionadduction, and $-31^{\circ}$ to $+12^{\circ}$ in internal-external rotation with applied loads from $150 \mathrm{~N}$ to $450 \mathrm{~N}$. The physiological data was compared to the actual motion and loads reproduced in the simulator to assess simulator performance. Commissioning trials included running the simulator for extended periods in excess of 100,000 cycles to assess reliability of the machine. During the commissioning trials, new JRI Orthopaedics Reverse VAIOS shoulder 42mm Cobalt Chrome Molybdenum (CoCrMo) against UHMWPE prostheses were fitted in all six simulator stations and tests were conducted in 50\% newborn calf serum (Sigma Aldrich n4637), giving a protein content of $26 \mathrm{~g} / \mathrm{L}$, at ambient temperature.

For the first wear test, 'Mug to Mouth' was selected as the ADL to use in the simulator at $1 \mathrm{~Hz}$. This ADL was selected because it has been used widely in shoulder biomechanical studies ${ }^{18,21,22,33-35}$. Prior to the wear test, the physiological data was compared to the actual motion and loads reproduced in the simulator to ensure the accuracy of the test. Loads applied over each cycle ranged between approximately $180 \mathrm{~N}$ to $250 \mathrm{~N}$ and the simulator applied load and physiological load cycles are shown in Figure 5. The root square mean error between the physiological load and actual simulator load was $9 \mathrm{~N}$. The physiological and simulator motion cycles are shown in Figure 6 . The root square mean error between the physiological and simulator position over a cycle is $3.3^{\circ}$ in flexion-extension, $1.4^{\circ}$ in abduction-adduction, and $2.0^{\circ}$ in 
internal-external rotation. A 2.0 million cycle wear test was performed with five articulating, and one static but loaded soak control, new 42mm JRI Orthopaedics Reverse VAIOS shoulder CoCrMo against UHMWPE prostheses tested in 50\% newborn calf serum (Sigma Aldrich n4637), giving an average protein content of 26 $\mathrm{g} / \mathrm{L}$, at ambient temperature. Gravimetric measurements were used (Denver Instruments TB-215D, $10 \mu \mathrm{g}$ ) to determine the polymeric wear. The gravimetric method employed was based on ISO 14242-2 for testing hip prostheses, in the absence of similar ISO protocol for shoulder prostheses, and volumetric wear was calculated using the density of the UHMWPE, which is $938 \mathrm{~kg} / \mathrm{m}^{3}$.

[Insert Figure 5.]

[Insert Figure 6.] 


\section{Results}

The commissioning trials and subsequent post-trial analysis of the simulator allowed the robustness of the machine to be assessed. No problems were observed with the frame and structure of the simulator, the pneumatic system and components, the electrical and electronic components, or the software. No problems were found with the flexion/extension drive train. Some of the bearings on the abduction/adduction drive train were found to be slightly notchy post-test. All of the bearings were replaced and periodic inspection and replacement as necessary implemented in the maintenance schedule. Regular lubrication of ball and socket joints on the internal/external rotation drive train was found to be required. Periodic inspection and a recommendation of replacing said joints, as appropriate, were added to the maintenance schedule.

The commissioning trials used motion and loading cycles for the ADL lifting a $0.5 \mathrm{Kg}$ mass to head height. In Figure 7 the physiological motion patterns and actual simulator motion patterns are shown for all three axes. The root square mean error between the physiological and simulator position over a cycle is $1.6^{\circ}$ in flexion-extension, $4.2^{\circ}$ in abduction-adduction, and $8.1^{\circ}$ in internal-external rotation.

\section{[Insert Figure 7.]}

The physiological load cycle and the simulator load cycle for the commissioning trials are shown in Figure 8. The root square mean error between the physiological and simulator loads is $18 \mathrm{~N}$ over a cycle. 


\section{[Insert Figure 8.]}

Figure 9 shows the wear results obtained from the test. Over 2.0 million cycles the average polymeric components wear rate was $14.2 \pm 2.1 \mathrm{~mm}^{3} / 10^{6}$ cycles.

[Insert Figure 9.] 


\section{Discussion}

Single station wear simulators have obvious limitations in the statistical relevance of the results. One such simulator ${ }^{12}$ had further limitations of using a constant load of $200 \mathrm{~N}$ and Ringer solution as the lubricant. Ringer solution is not a lubricant recommended by ISO 14242-1, and water based lubricants have been shown to generate transfer films when wear testing in a simulator ${ }^{\mathbf{3 6}}$. Another single-station simulator only applied motion in one axis, abduction-adduction, and gave inconsistent results in two wear studies $^{\mathbf{1 3 , 1 4}}$. Such simplification of motion to only one axis has been shown on hip simulators to fundamentally change the wear of the prostheses and to produce results not comparable to those when subject to multi-axis motion ${ }^{\mathbf{1 8 , 1 9}}$. A single station machine that used two axes of motion, running at $0.8 \mathrm{~Hz}$, could not measure wear gravimetrically and as such acknowledged wear assessment as problematic ${ }^{15}$.

Multi-station simulators have been used to test shoulder prostheses, but to date all are hip ${ }^{\mathbf{9}, 11}$ or knee simulators ${ }^{10}$, baring one of unspecified type ${ }^{37}$ which is most probably a knee simulator as translational motion was applied. Table 1 shows these multi-station shoulder tests of CoCrMo against UHMWPE tested in simulators and the various test conditions and results. This study is included in Table 1 for comparison. As can be seen from the table, the studies using hip and knee simulators have used nonphysiological loading, applying either sinusoidal or constant loading. The applied motions also vary considerably and do not correspond to shoulder ADLs identified and described elsewhere ${ }^{\mathbf{8 , 2 2}}$. 


\section{[Insert Table 1.]}

A study of the shoulder performing typical $\mathrm{ADLs}^{8}$ measured ranges of shoulder flexion/extension of $97^{\circ}$, abduction/adduction of $60^{\circ}$ and internal/external rotation of $67^{\circ}$. It is worth noting that the study was of a small number of ostensibly healthy male subjects. Further studies providing kinematic information on male and female subjects with and without shoulder prostheses so that all of the necessary ADLs could be simulated would be extremely useful. ISO14242-1 defines the gait cycle for hip simulator testing with ranges of $43^{\circ}$ in flexion-extension, $11^{\circ}$ in abduction-adduction axis and $12^{\circ}$ in internal-external rotation. Knee simulators ${ }^{\mathbf{2 7}, 31}$ typically apply ranges of motion up to $65^{\circ}$ in flexion-extension, $12^{\circ}$ in abduction-adduction axis and $10^{\circ}$ in internal-external rotation. As hip and knee simulators are designed to replicate the gait cycle, whilst not impossible, it is unlikely that most hip and knee simulators are physically capable of the ranges of motion necessary to apply a full range of shoulder ADLs. An opportunity therefore existed to design a multi-station shoulder simulator capable of testing with physiological motion patterns in three axes and with physiological loading.

The Newcastle Shoulder Wear Simulator has five articulating stations with three axes of motion and applies physiological motion and loading. The simulator is capable of $110^{\circ}$ of motion in flexion-extension, $90^{\circ}$ in abduction-adduction axis and $90^{\circ}$ in internalexternal rotation. Therefore, the Newcastle Shoulder Wear Simulator has ranges of available motion in all three axes to accommodate recognised physiological patterns of shoulder motion when performing ADL's. 
The commissioning trials, and indeed subsequent wear test, proved that the Newcastle Shoulder Wear Simulator was robust and reliable. Reliability was observed not only in the ability of the simulator to function as intended between routine maintenance but also to maintain the motion and loading simulation over the course of testing for millions of cycles.

With any simulator questions may be asked over differences between requested and applied motions and loads. Many factors can cause these discrepancies. The friction of the joints under test will change over the course of the test as they wear, as will the collective friction of all the bearings in the simulator. The viscosity of the serum may change from when it is new to when it requires replacement a few days later. Small amounts of flexing of the drive systems components, or indeed the frame may be occurring. Hysterisis will also be evident as the simulator continually examines its actual position to requested position and tries to correct for any errors.

In the first wear test of the Newcastle Shoulder Wear Simulator, the 'Mug to Mouth' ADL was applied to $42 \mathrm{~mm}$ reverse shoulder prostheses. The wear test result of 14.2 $\mathrm{mm}^{3} / 10^{6}$ cycles in this study might initially appear to compare favourably with reverse shoulder prostheses tested in a hip simulator by Kohut et al $\mathbf{l}^{\mathbf{1 0}}$ who measured a wear rate of $14.0 \mathrm{~mm}^{3} / 10^{6}$ cycles. However, we must consider the various differences in test conditions before drawing comparison.

Analysing wear tracks and wear factors in detail is beyond the scope of this study, however, consideration and comparison of the load applied and the size of the 
prostheses is appropriate. CoCrMo joints articulating against UHMWPE can be expected to be operating in a mixed lubrication regime ${ }^{38,39}$, albeit one in which the vast majority of the load across the joint is supported by asperity contact as in a boundary lubrication regime. The Lancaster ${ }^{\mathbf{4 0}}$ wear equation is applicable to the wear of joints operating in a boundary lubrication regime:

$$
V=k P x
$$

where $V$, the volume of material removed by wear, is proportional to the product of wear factor $k$, applied load $P$ and sliding distance $x$. Therefore as applied load increases, wear can be expected to increase proportionally. As joint size increases, wear rate should also increase as sliding distance increases. However, this effect is countered slightly by the subsequent increase in entraining velocity generating partial fluid film lubrication wherein some of the load across the joint is supported by the fluid in the joint rather than asperity contact. A predicted wear rate can be calculated using the wear rate found in this study and adjusted for load and prosthesis diameter differences and then a comparison made with measured wear rate in other studies. For example, the load applied by Kohut et al ${ }^{10}$ was $756 \mathrm{~N}$ and the joints were of a smaller $36 \mathrm{~mm}$ diameter to the $42 \mathrm{~mm}$ diameter in this study;

$14.2 \mathrm{~mm}^{3} / 10^{6}$ cycles $x(756 \mathrm{~N} / 250 \mathrm{~N}) \times(36 \mathrm{~mm} / 42 \mathrm{~mm})=36 \mathrm{~mm}^{3} / 10^{6}$ cycles. 
Allowing for load and joint diameter differences, the expected wear rate from the Kohut et al study would be $36 \mathrm{~mm}^{3} / 10^{6}$ cycles and therefore $250 \%$ greater than the measured $14.3 \mathrm{~mm}^{3} / 10^{6}$ cycles.

Wirth et al ${ }^{\mathbf{1 1}}$ used a knee simulator to apply three axes of motion and a constant load of $756 \mathrm{~N}$ to $48 \mathrm{~mm}$ diameter CoCrMo joints. The authors recognized that high constant loading for extended periods was unusual in the shoulder. Making a comparison to this study allowing for triple the applied load and the larger diameter joints, a wear rate of approximately $49 \mathrm{~mm}^{3} / 10^{6}$ cycles might be expected. The measured wear rate by Wirth et al was $49.4 \mathrm{~mm}^{3} / 10^{6}$ cycles, and the two studies therefore show remarkable agreement.

Vaupel et $a l^{9}$ used a modified hip simulator with only two axes of motion and applied sinusoidal loading alternating between two axes every 250,000 cycles. The load was approximately triple the applied load of this study and smaller $36 \mathrm{~mm}$ diameter glenospheres were used. Based on these differences, a wear rate of $36 \mathrm{~mm}^{3} / 10^{6}$ cycles would be expected compared to this study. At $126 \mathrm{~mm}^{3} / 10^{6}$ cycles, the recorded result was $350 \%$ greater.

The McNulty et al ${ }^{37}$ study does not specify the type of simulator used, however, as translation is applied it is probably a knee type simulator. Unfortunately, the lack of information on applied motion and load, makes comparison and comment rather tenuous. Nevertheless, using the average human body weight for North America measured during the period the study was published ${ }^{41}$ of $80.7 \mathrm{Kg}$, a wear rate of 
approximately $51 \mathrm{~mm}^{3} / 10^{6}$ cycles would be expected. This compares to a measured result of $49.8 \mathrm{~mm}^{3} / 10^{6}$ cycles, assuming the same density of UHMWPE as used in this study. Whilst this shows good agreement, this observation must be tempered by the lack of data available on the McNulty et al study.

Drawing comparison of this study with the published shoulder studies using simulators not specifically designed to wear test shoulders shows good agreement with two of the published results when adjusted for loading and joint size. Conversely, poor agreement is seen with the other two results. Due to the various differences in motion and loading patterns and ranges, this is perhaps unsurprising. Therefore, to gain more confidence in the wear result of this study using a bespoke engineered shoulder wear simulator and using the motion and load patterns of a recognised ADL, other simulator wear studies must be considered.

In vitro data is available for CoCrMo against UHMWPE tested in diluted bovine serum from hip simulator studies is shown in Table 2, with this study included for comparison. It can be seen that the loading in the hip simulator studies is substantially greater than in this study and the gait cycle motion is applied only in two axes. Using the wear results from this study and making comparison to the hip simulator studies accounting for joint diameter and peak loading differences, the predicted wear rates would be approximately double those measured.

[Insert Table 2.] 
The reason for this discrepancy may be attributable to differences in the wear path and loading between the shoulder 'Mug to Mouth' ADL and hip gait cycles. In particular, during the swing phase of the gait cycle a combination of increased entraining velocity and low load may be responsible for low wear during this phase. Table 3 shows further analysis of these hip simulator studies using an estimated mean load rather than peak load, and swing phase duration during which wear was assumed to be minimal. The predicted wear rates using this data show good agreement with the hip simulator studies measured wear rates.

[Insert Table 3.]

\section{Conclusion}

The Newcastle Shoulder Wear simulator is a multi-station simulator engineered with ranges of motion sufficiently large to accommodate recognized shoulder ADLs and capable of applying physiological patterns of motion and load. Prior to this study, to the authors' best knowledge, no shoulder simulator wear studies had fully replicated the physiological motion and loading of a typical shoulder ADL. Comparison of the wear rate from this study with hip simulator studies shows good agreement when accounting for the differences in prostheses diameter and test conditions of the gait cycle versus the 'Mug to Mouth' shoulder ADL. The wear result obtained in this study can form the basis of in vitro wear knowledge of shoulder prostheses tested using recognized shoulder ADLs, which are possible with this simulator. 



\section{Acknowledgements}

The authors would like to acknowledge the financial support of the Leverhulme Trust for this research project. Additional gratitude is extended to the Engineering and Physical Sciences Research Council (EPSRC). JRI Orthopaedics kindly supplied the sample VAIOS prostheses free of charge.

\section{Declaration of conflicting interests}

The authors declare that there is no conflict of interest.

\section{Funding}

This research was generously funded by the Leverhulme Trust, grant reference: F/00 125/Z, and the Engineering and Physical Sciences Research Council (EPSRC) Newcastle University Impact Award: EP/J014915/1. 


\section{References}

1. Willert, H.G., Bertram, H., Buchhorn, G. H. (1989) Osteolysis in alloarthroplasty of the hip. Clinical Orthopaedics and Related Research, 258, 95-107.

2. Schmalzried, T. P., Jasty, M., Rosenberg, A., Harris, W. H., (1992) Periprosthetic bone loss in total hip arthroplasty. Journal of Bone and Joint Surgery, 74-A, 6, 849-863.

3. Illgen Ii, R.L., Bauer, L.M., Hotujec, B.T., Kolpin, S.E., Bakhtiar, A., Forsythe, T.M., (2009) Highly Crosslinked Vs Conventional Polyethylene Particles: Relative In Vivo Inflammatory Response. The Journal of Arthroplasty, 24, 117-124.

4. Scarlat M M, Matsen F A. (2001) Observations on retrieved polyethylene glenoid components. Journal of Arthroplasty, 2001; 16(6):795-801.

\section{Wirth M A, Agrawal C M, Mabrey J D, Dean D D, Blanchard C R, Miller} M A et al. (1999) Isolation and characterization of polyethylene wear debris associated with osteolysis following total shoulder arthroplasty. Journal of Bone and Joint Surgery Am, 81(1): 95-100.

6. No_Authors_Listed. (2014) National Joint Registry for England, Wales and Northern Ireland. 11th Annual Report.

7. No_Authors_Listed. (2013) National Joint Registry for England, Wales and Northern Ireland. 10th Annual Report.

8. Murray, I.A., Johnson, G.R. (2004) A study of the external forces and moments at the shoulder and elbow while performing every day tasks. Clinical Biomechanics, 19, 586-594. 
9. Vaupel, Z.M., Baker, K.C., Kurdziel, M.D., Wiater, J.M., (2012) Wear simulation of reverse total shoulder arthroplasty systems: effect of glenosphere design. Journal of Shoulder and Elbow Surgery, 21, 1422-1429.

10. Kohut, G., Dallmann, F., Irlenbusch, U. (2012). Wear-induced loss of mass in reversed total shoulder arthroplasty with conventional and inverted bearing materials. Journal of Biomechanics, 45, 469-473.

11. Wirth, M. A., Klotz, C., Deffenbaugh, D. L., McNulty, D., Richards, L., Tipper, J. L. (2009) Cross-linked glenoid prosthesis: A wear comparison to conventional glenoid prosthesis with wear particulate analysis. Journal of Shoulder and Elbow Surgery, 18, 130-137.

12. Muller, D., Marti, A., Herzig, P., (2001) Year Evaluation of PEEK for shoulder endoprosthetics in a screening and a simulated physiological wear test. In 47th Annual Meeting, Orthopaedic Research Society. San Francisco, California.

13. Pijl, A.J., Swieszkowski, W., Bersee, H.E.N., (2004) Technical Application Series. Design of a wear simulator for in vitro shoulder prostheses testing. Experimental Techniques, 28, 45-48.

14. Swieszkowski, W., Bersee, H.E.N., Kurzydlowski, K.J., (2011) Effect of the design parameters on the in vitro wear performance of total shoulder arthroplasties. Materials Science and Engineering: C, 31, 313-319.

15. Geary, C., O'Donnell, G.E., Jones, E., FitzPatrick, D., Birkinshaw, C., (2010) Automated In-Vitro Testing of Orthopaedic Implants: A Case Study in Shoulder Joint Replacement. Proceedings of the Institution of Mechanical Engineers, Part H: Journal of Engineering in Medicine, 224, 1297-1309. 
16. ISO14242-1. (2000). Implants for surgery - wear of total hip joint prostheses, part 1. Loading and displacement parameters for wear-testing machines and corresponding environmental conditions for test. ISO, Geneva.

17. Paul, J.P. (1967) Forces Transmitted by Joints in the Human Body. Engineering in Medicine., 181, 37, 8-15.

18. Smith, S. L., Unsworth, A. (2000) Simplified motion and loading compared to physiological motion and loading in a hip joint simulator. Proceedings of the Institution of Mechanical Engineers, Part H (Engineering in Medicine), 214, H3, 233-238.

19. Barbour, P. S. M., Stone, M. H., Fisher, J. (1999) A hip joint simulator study using simplified loading and motion cycles generating physiological wear paths and rates. Proceedings of the Institution of Mechanical Engineers, Part $H$ (Engineering in Medicine), 213, 455-467.

20. ISO14242-2. (2000) Implants for surgery - wear of total hip joint prostheses, part 2. Methods of measurement. ISO, Geneva.

21. Charlton, I.W., Johnson, G.R. (2006) A model for the prediction of the forces at the glenohumeral joint. Proceedings of the I MECH E Part H Journal of Engineering in Medicine, 220, 801-812.

22. Kontaxis, A., (2010) Biomechanical analysis of reverse anatomy shoulder prosthesis. Newcastle.

23. Liao, Y.S., McKellop, H.A., Lu, Z., Campbell, P., Benya, P., (2003) The effect of frictional heating and forced cooling on the serum lubricant and wear of UHMW polyethylene cups against cobalt-chromium and zirconia balls.

Biomaterials, 24, 3047-3059. 
24. Saikko, V.,(2005) A hip wear simulator with 100 test stations. Proceedings of the Institution of Mechanical Engineers. Part H, Journal of Engineering in Medicine, 219, 309-318.

25. Dowson, D., Jobbins, B. (1988) Design and development of a versatile hip joint simulator and a preliminary assessment of the wear and creep in Charnley total replacement hip joints. Eng. Med., 17, 3, 111-117.

26. Clarke, I. C., Good, V., Anissian, L., Gustafson, A. (1997) Charnley wear model for validation of hip simulators - ball diameter versus polytetrafluoroethylene and polyethylene wear. Proceedings of the Institution of Mechanical Engineers, Part H (Engineering in Medicine), 211, H1, 25-36.

27. Burgess, I. C., Kolar, M., Cunningham, J. L., Unsworth, A. (1997) Development of a six station knee wear simulator and preliminary wear results. Proceedings of the Institution of Mechanical Engineers, Part H (Engineering in Medicine), 211, 1, 37-47.

28. Smith, S. L., Burgess, I. C., Unsworth, A. (1999) Evaluation of a hip joint simulator. Proceedings of the Institution of Mechanical Engineers, Part H (Engineering in Medicine), 213, H6, 469-473.

29. Goldsmith, A. A. J., Dowson, D. (1999) A multi-station hip joint simulator study of the performance of 22mm diameter zirconia - UHMWPE total replacement joints. Proceedings of the Institution of Mechanical Engineers, Part H (Engineering in Medicine), 213, H2, 77-90.

30. Smith, S. L., Unsworth, A. (2001) A 5 station hip joint simulator. Proceedings of the Institution of Mechanical Engineers, Part H(Engineering in Medicine), 215, H1, 61-64. 
31. Barnett, P.I., McEwen, H.M.J., Auger, D.D., Stone, M.H., Ingham, E., Fisher, J. (2002) Investigation of wear of knee prostheses in a new displacement/force-controlled simulator. Proceedings of the Institution of Mechanical Engineers, Part H (Engineering in Medicine), 216, 51-61.

32. Joyce, T. J., Unsworth, A. (2000) The design of a finger wear simulator and preliminary results. Proceedings of the Institution of Mechanical Engineers, Part H (Engineering in Medicine), 214, 519-526.

33. Kontaxis, A., Johnson, G.R., (2009). The biomechanics of reverse anatomy shoulder replacement - A modelling study. Clinical Biomechanics, 24, 254-260.

34. Masjedi, M., Johnson, G.R., (2010). Glenohumeral contact forces in reversed anatomy shoulder replacement. Journal of Biomechanics, 43, 2493-2500.

35. Murray, I.A., Johnson, G.R., (2004). A study of the external forces and moments at the shoulder and elbow while performing every day tasks. Clinical Biomechanics, 19, 586-594.

36. Good, V., Clarke, I., Anissian. (1996) Water and bovine serum lubrication compared in simulator PTFE/CoCr wear model. J. of Biomedical Materials Research (Applied Biomaterials), 33, 275-283.

37. McNulty, D.E., Swope, S.W., Deffenbaugh, D. (2005) In-vitro Wear Testing Of Total Shoulder Prostheses Using Conventional And Crosslinked Polyethylene. Society For Biomaterials 30th Annual Meeting Transactions, Memphis, 701-703.

38. O’Kelly, .J, Unsworth, A., Dowson, D., Wright, V. (1979) An experimental study of friction and lubrication in hip prostheses. Engineering in Medicine, 8, 3, 153-159. 
39. Unsworth, A., Scholes, S.C., Smith, S.L., Elfick, A.P.D., Ash, H.A. (2000) Tribology of Replacement Hip Joints. Tribology Series, 38, 195-202.

40. Lancaster, J.K., (1973) Dry bearings: a survey of materials and factors affecting their performance. Tribology, 6, 219-251.

41. Walpole, S.C., Prieto-Merino, D., Edwards, P., Cleland, J., Stevens, G., Roberts, I. (2012) The weight of nations: an estimation of adult human biomass. BMC Public Health, 12 (1), 439.

42. Barbour, P. S. M., Stone, M. H., Fisher, J. (2000) A hip joint simulator study using new and physiologically scratched femoral heads with ultra-high molecular weight polyethylene acetabular cups. Proceedings of the Institution of Mechanical Engineers, Part H (Engineering in Medicine), 214, 569-576.

43. Smith S L, Unsworth A. (1999) A comparison between gravimetric and volumetric techniques of wear measurement of UHMWPE acetabular cups against zirconia and CoCrMo femoral heads in a hip simulator. Proceedings of the Institution of Mechanical Engineers, Part H (Engineering in Medicine), 213, H6, 475-483.

44. Wang, A., Stark, C., Dumbleton, J. H. (1996) Mechanistic and morphological origins of ultra-high molecular weight polyethylene wear debris in total joint replacement prostheses. . Proceedings of the Institution of Mechanical Engineers, Part H (Engineering in Medicine), 210, 141-155.

\section{Good, V.D., Clarke, I.C., Gustafson, G.A., Downs, B., Anissian, L.,} Sorenson, K., (2000) Wear of ultra-high molecular weight polyethylene and polytetrafluoroethylene in a hip simulator. Acta Orthopaedica Scandinavica, 71, 365-369. 